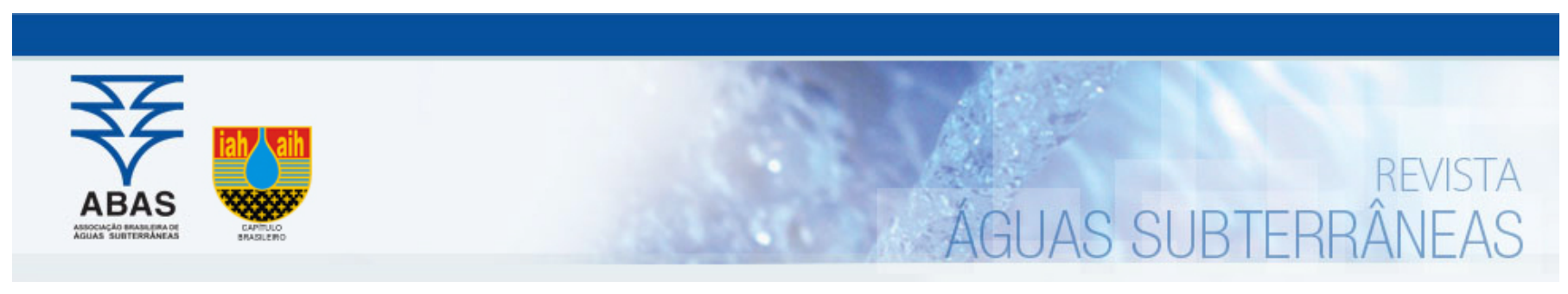

Artigos

\title{
Transporte de solutos em diferentes cenários geológicos gerados por modelos estocásticos de cadeias de Markov
}

\section{Solute transport in different geological scenarios generated by stochastic model of Markov chains}

\author{
Elias Hideo Teramoto $^{1}$; Hung Kiang Chang ${ }^{2}$; Maria Rita Caetano-Chang ${ }^{\mathbf{凶}}$ \\ 1 Centro de Estudos Ambientais (UNESP), Campus de Rio Claro / Lebac - Laboratório de Estudo de Bacias (UNESP), Campus de Rio \\ Claro, São Paulo, Brasil. \\ 2 Departamento de Geologia Aplicada (UNESP), Campus de Rio Claro / Lebac - Laboratório de Estudo de Bacias (UNESP), Campus de \\ Rio Claro, São Paulo, Brasil. \\ 3 Departamento de Geologia Aplicada (UNESP), Campus de Rio Claro, São Paulo, Brasil.
}

$\triangle$ teramoto@rc.unesp.br; chang@rc.unesp.br; mrchang@rc.unesp.br

\begin{tabular}{|c|c|}
\hline & Resumo \\
\hline $\begin{array}{l}\text { Palavras-chave: } \\
\text { Heterogeneidade geológica. } \\
\text { Modelos estocásticos. } \\
\text { Conectividade entre corpos arenosos. }\end{array}$ & $\begin{array}{l}\text { O entendimento do comportamento de contaminantes em subsuperfície é crucial para quantificação do risco à saúde humana } \\
\text { e planejamento dos sistemas de remediação. Por sua vez, a confiabilidade das previsões do comportamento da pluma conta- } \\
\text { minante está relacionada à variabilidade litológica em subsuperfície, de forma que é elevado o grau de incerteza em aquíferos } \\
\text { heterogêneos. O Aquífero Rio Claro, na região do município de Paulínia (SP), apresenta marcante heterogeneidade litológica. } \\
\text { Com vistas a investigar o efeito dessa heterogeneidade no transporte e destino de contaminantes dissolvidos em águas subter- } \\
\text { râneas, foram realizadas simulações considerando diferentes cenários geológicos nesse aquífero. A partir de informações lito- } \\
\text { lógicas provenientes de } 58 \text { descrições de sondagem, foram gerados } 10 \text { cenários geológicos distintos empregando-se o método } \\
\text { estocástico de Cadeias de Markov. Cada cenário geológico foi inserido dentro de modelos numéricos para avaliação do trans- } \\
\text { porte de contaminantes dissolvidos em águas subterrâneas. Os resultados indicam ampla variabilidade das dimensões das } \\
\text { plumas dissolvidas, bem como das direções de migração; o principal fator responsável por tal variação é o grau de conectividade } \\
\text { dos corpos arenosos dotados de elevada permeabilidade. Mediante esses resultados, constata-se que as incertezas intrínsecas } \\
\text { à conectividade dos corpos arenosos dificultam a previsão de migração de contaminantes dissolvidos e o planejamento dos } \\
\text { sistemas de remediação em casos de aquíferos análogos ao Rio Claro. }\end{array}$ \\
\hline
\end{tabular}

Abstract

Keywords:

Geological heterogeneity.

Stochastic models.

The understanding of subsurface contaminants behavior is critical for the quantification of human health risk and planning of active remediation. Since the prediction reliability of plume behavior is related to lithological variability in subsurface, there is a high degree of uncertainties in heterogeneous aquifers. In order to investigate the effect of observed heterogeneity of Rio Claro Aquifer, located in the municipality of Paulínia(SP), on the fate and transport of dissolved contaminants in groundwater, simulations encompassing different geological scenarios was carried out. Based on lithological information from 58 survey description, 10 different geological scenarios were generated using the stochastic model of Markov Chain. Each geological scenario was reproduced into numerical models to evaluate the transport of contaminants dissolved in groundwater. The results indicate wide variability of the dimensions and migration directions of the dissolved plumes. The main factor responsible for such variation is the degree of connectivity of sandy bodies with high permeability. These results suggest that uncertainties are intrinsically related to the sandy bodies connectivity, hampering the prediction of contaminant plumes migration and the planning of the remediation systems in similar aquifers to Rio Claro.

DOI: http://dx.doi.org/10.14295/ras.v31i4.28860

\section{INTRODUÇÃO}

Modelos matemáticos são ferramentas essenciais no estudo de áreas contaminadas, uma vez que permitem previsões do comportamento de contaminantes, quantificação do risco à saúde humana e planejamento dos sistemas de remediação a serem implantados. Entretanto, modelos determinísticos são incapazes de representar adequadamente a variabilidade espacial dos valores de condutividade hidráulica em aquíferos fortemente heterogêneos, agregando um elevado grau de incerteza aos resultados obtidos.

Em oposição aos modelos determinísticos, incapazes de incorporar incertezas relacionadas às complexas heterogeneidades geológicas, a abordagem estocástica emergiu, a partir da década de 1970, como um promissor campo destinado à modelagem de fluxo e transporte de solutos (GELHAR, 1986). Tendo em vista que as heterogeneidades geológicas são intrinsecamente 
relacionadas à arquitetura de fácies sedimentares, os padrões de distribuição das propriedades do aquífero podem ser simulados a partir da distribuição dessas fácies, baseando-se em modelos que empregam as Cadeias de Markov. Esta técnica estatística simples é utilizada para caracterização de processos recorrentes no espaço ou no tempo, como a sucessão de fácies em um mesmo contexto deposicional (KRUMBEIN \& DACEY,1969).

Apoiando-se na premissa de que as sequências estratigráficas possuem propriedades markovianas, é possível determinar a probabilidade de transição entre litologias dentro de um sistema deposicional.

Embora os modelos estocásticos, incluindo o de Cadeias de Markov, sejam amplamente empregados para gerar diferentes situações com a distribuição espacial da condutividade hidráulica, especial atenção tem sido destinada à avaliação da interconectividade de fácies permeáveis. Moreno \& Tsang (1994), Koltermann \& Gorelick (1996), Maxwell et al. (2008), Zhang et al. (2013), Fogg \& Zhang (2016), Tyukhova \& Willmann (2016), dentre outros, têm argumentado que em meios heterogêneos a continuidade e a interconectividade das fácies arenosas, dotadas de elevada permeabilidade, exercem controle mais efetivo no fluxo e transporte de solutos que a distribuição randômica da condutividade hidráulica. A conectividade de corpos arenosos pode ter forte impacto na migração de solutos, uma vez que lentes permeáveis contínuas representam vias preferenciais para um movimento advectivo mais eficiente. 0 efeito da continuidade espacial das lentes permeáveis no transporte de solutos é conhecido como canalização, sendo descrito por diversos auto- res, incluindo Fogg (1986), Koltermann \& Gorelick (1996), Maxwell et al. (2008), Bianchi et al. (2011), Zhang et al. (2013) e Fogg \& Zhang (2016).

Dada a vulnerabilidade de aquíferos rasos, o entendimento do comportamento dos contaminantes em subsuperfície é de extrema importância, particularmente em áreas de pólos petroquímicos como é o caso de Paulínia (SP), onde o Aquífero Rio Claro se estende por vasta área. O Aquífero Rio Claro é aí representado por litologias diversas, depositadas em ambiente fluvial meandrante (FERNANDES, 1997). Este contexto deposicional é responsável pela forte heterogeneidade do aquífero (TERAMOTO, 2015), fato que contribui para o elevado grau de incerteza quanto à distribuição espacial das propriedades do aquífero e, por consequência, quanto ao comportamento de contaminantes dissolvidos na água subterrânea. Como descrito por Fernandes (1997), nessa região estão presentes litotipos pertencentes ao Embasamento Cristalino (granitos, gnaisses, xistos etc.; Precambriano), a unidades litoestratigráficas da Bacia do Paraná - Subgrupo Itararé (arenitos, diamictitos, siltitos, lamitos etc.; Permiano-Carbonífero), Formação Serra Geral (intrusivas básicas; Cretácio Inferior), Formação Rio Claro (arenitos, siltitos, lamitos; Cenozoico) - e a depósitos quaternários (Figura 1).

Utilizando-se de dados de sondagens realizadas no Aquífero Rio Claro em Paulínia (Figura 1), o presente trabalho analisou o comportamento do transporte de solutos em diferentes cenários geológicos, gerados por simulações estocásticas do modelo de Cadeias de Markov, com especial enfoque na avaliação da influência relativa da continuidade espacial das fácies arenosas.

Figura 1 - Mapa geológico da região de Paulínia (FERNANDES, 1997) e localização da área de estudo

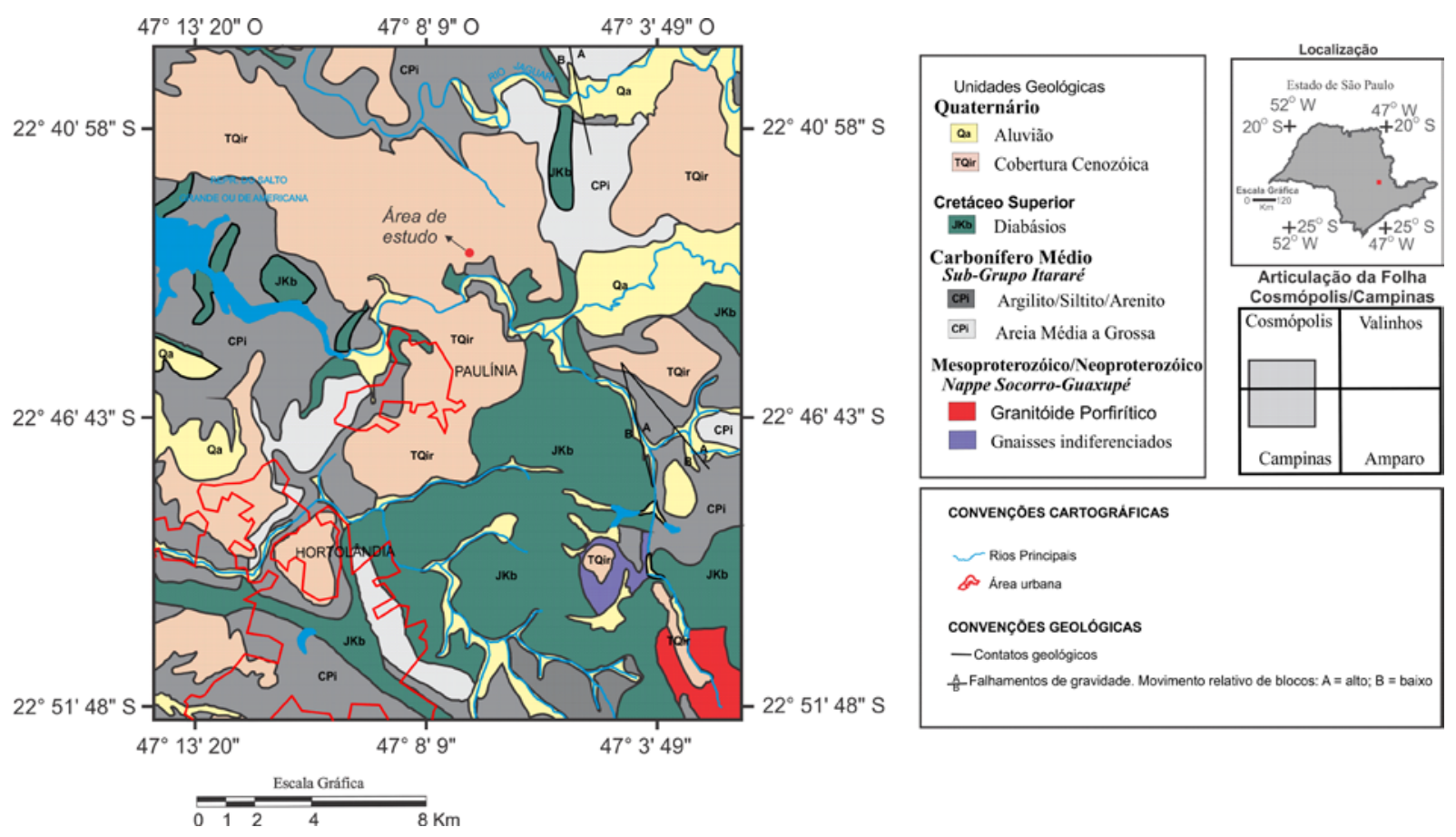




\section{MATERIAIS E MÉTODOS}

\subsection{Constituição do Aquífero Rio Claro}

Com base nas descrições de Fernandes (1997) e Melo (1995), bem como em trabalhos de campo de Teramoto (2015) nas circunvizinhanças da área de estudo, foram definidas as principais fácies sedimentares e respectivas constituições litológicas que compõem o Aquífero Rio Claro.

\section{Litotipos arenosos}

Compreende arenitos médios a grossos que passam lateralmente para arenitos argilosos. São friáveis, textural e mineralogicamente imaturos, normalmente com presença de seixos subangulares de quartzo e de siltitos laterizados. Conforme Teramoto (2015), os arenitos apresentam localmente continuidade espacial e espessuras variando de alguns centímetros até $3 \mathrm{~m}$; ocorrem em diferentes profundidades, alternando-se com litotipos pelíticos. Segundo esse autor, constituem fácies de canais fluviais.

\section{Litotipos pelíticos}

Constituem sedimentos pelíticos de textura variável, podendo ser levemente arenosos. Segundo Teramoto (2015), suas características indicam tratar-se das fácies $\mathrm{Si}$, Ag e Laf de Fernandes (1997) e Trcm de Melo (1995). Teramoto (2015) afirma que as litologias pertencentes às fácies de planície de inundação constituem os litotipos predominantes na área de estudo, particularmente os siltitos argilosos. Em afloramentos, apresentam geralmente aspecto maciço, por vezes intensamente fraturado. São majoritariamente lamitos, argilitos arenosos e siltitos argilosos, por vezes exibindo fósseis vegetais. Conforme Teramoto (2015), as espessuras das camadas pelíticas variam de alguns centímetros a mais de 5 metros, alternando-se com litotipos arenosos.

\subsection{Compilação de informações litológicas}

Os dados foram obtidos a partir de 58 sondagens à percussão (SPT) realizadas na área. A utilização desses dados em detrimento dos perfis descritivos dos poços de monitoramento se justifica pelo fato de as SPT atingirem porções mais profundas de subsuperfície e apresentarem maior homogeneidade na descrição dos perfis litológicos obtidos a partir de sondagens à percussão (SPT). Esses perfis foram cedidos pelas empresas que executaram os ensaios.

\subsection{Associação entre litotipos e condutividade hidráulica}

A caracterização hidráulica na área de estudo e áreas contíguas foi efetuada a partir de ensaios de slug, que permitiram mensurar valores de condutividade hidráulica nas porções adjacentes à seção filtrante dos poços de monitoramento. Desse modo, a partir da identificação dos litotipos em subsuperfície e conhecendo-se o perfil construtivo do poço de monitoramento, foi possível atribuir valores de condutividade hidráulica a litotipos específicos. A Tabela 1 apresenta os litotipos e respectivos intervalos de valores de condutividade hidráulica obtidos em campo, bem como o número de ensaios realizados para obtenção desse parâmetro, extraídos do trabalho de Teramoto (2015).

Tabela 1 - Litotipos presentes na área de estudo e respectivos valores médios de condutividade hidráulica (TERAMOTO, 2015)

\begin{tabular}{ccccc}
\hline Litotipo & $\mathrm{K}(\mathrm{m} / \mathrm{s})$ mínimo & $\mathrm{K}(\mathrm{m} / \mathrm{s})$ médio & $\begin{array}{c}\mathrm{K}(\mathrm{m} / \mathrm{s}) \text { má- } \\
\text { ximo }\end{array}$ & $\begin{array}{c}\text { Número de } \\
\text { ensaios }\end{array}$ \\
\hline Siltito argiloso & $7,39 \times 10^{-8}$ & $9,47 \times 10^{-7}$ & $6,64 \times 10^{-6}$ & 57 \\
Argilito arenoso & $7,20 \times 10^{-6}$ & $1,91 \times 10^{-5}$ & $3,82 \times 10^{-5}$ & 31 \\
Arenito médio argiloso & $5,23 \times 10^{-5}$ & $7,27 \times 10^{-5}$ & $8,98 \times 10^{-5}$ & 5 \\
Arenito médio a grosso micro- & $1,03 \times 10^{-4}$ & $2,36 \times 10^{-4}$ & $6,72 \times 10^{-4}$ & 23 \\
conglomerático & & & &
\end{tabular}

\subsection{Simulação das heterogeneidades geológicas - T-PROGS}

O aplicativo T-PROGS (CARLE \& FOGG, 1997) foi empregado para modelar a variabilidade geológica tridimensional da área estudada, utilizando informações dos perfis litológicos obtidos pelas sondagens a percussão. O T-PROGS realiza simulações estocásticas com base na probabilidade de transição de fácies, que podem ser modeladas pelas Cadeias de Markov. A probabilidade de transição define simplesmente que a probabilidade da litologia $k$ ocorrer na localização $x+h$ depende tão somente da litologia presente na posição x. 0 modelo de Cadeia de Markov define a função que relaciona a probabilidade de transição $\mathrm{t}_{\mathrm{jk}}(\mathrm{h})$ em resposta ao incremento de $h$, conforme a equação (1):

$$
t_{j k}(h)=\operatorname{Pr}\{k \text { ocorrer em } x+h \mid j \text { ocorrer em } x\}
$$

Os coeficientes da exponencial $r_{j k}$ são os parâmetros a serem ajustados, sendo conhecidos como taxas condicionais de mudança da litologia j para a litologia $k$, por unidade de comprimento do passo de distância $\mathrm{h}$.

A probabilidade de transição é oriunda da equação (2) de probabilidade condicional:

$$
\operatorname{Pr}\left\{B^{\prime} \mid A\right\}=\frac{\operatorname{Pr}\left\{A \text { e } B^{\prime}\right\}}{\operatorname{Pr}\{A\}}
$$

Onde, A representa $\{j$ ocorrer em $\mathrm{x}\}$ e B' representa $\{k$ ocorrer em $x+h$.

As taxas de transição são ajustadas para garantir a melhor correlação entre o modelo de Cadeias de Markov e os dados de 
probabilidade de transição observados. Uma vez que as Cadeias de Markov foram desenvolvidas para simular as transições de fácies na direção vertical, um modelo de variabilidade espacial é aplicado para simular as transições nas direções x e y, estabelecendo-se estimativas da razão do comprimento médio nas direções x e y em relação à direção vertical (z).

\subsection{Cenário hipotético de contaminação}

A seleção da área de estudo teve como critério a ocorrência de fortes heterogeneidades litológicas, o que dificulta a previsão da migração de contaminantes por intermédio de modelos numéricos de fluxo e transporte. Foi então idealizado um cenário hipotético de contaminação, com vistas a reproduzir a migração de contaminantes no aquífero analisado.

A área-fonte foi admitida como portadora de um volume de fase líquida não aquosa na forma residual, situado entre $5 \mathrm{~m}$ e $6 \mathrm{~m}$ de profundidade. Como demonstrado por Teramoto \& Chang (2017), a solubilização da fase líquida não-aquosa (NAPL) em área contígua à do presente estudo é resultado de inúmeros fatores, tais como velocidade de fluxo, taxa de biodegradação, saturação da fase não aquosa e da flutuação do nível d'água. Entretanto, para simplificação do modelo, admitiu-se a existência de equilíbrio termodinâmico entre a água e o NAPL no poro, baseando-se na Lei de Raoult (BANERJEE, 1984), com a fixação de uma concentração na porção compreendida pela área-fonte idealizada. No presente caso, para avaliar cenários conservadores foi admitido que o contaminante dissolvido na fase aquosa não está sujeito à perda de massa por biodegradação.

\subsection{Simulações de fluxo e transporte}

Os diferentes cenários geológicos tridimensionais gerados pelo T-PROGS foram convertidos para valores de condutividade hidráulica e porosidade específica equivalente, que foram empregados como parâmetros de entrada para o aplicativo Visual Modflow; esse aplicativo simula o fluxo por intermédio do Método de Diferenças Finitas com bloco centrado (HARBAUGH, 2005).

O domínio do modelo empregado nas simulações é representado por um volume com $500 \mathrm{~m}$ de comprimento, $400 \mathrm{~m}$ de largura e 20 m de espessura (Figura 2), e foi discretizado em 250 colunas, 200 linhas e 20 camadas. As condições de contorno empregadas foram de carga especificada (Condição de Contorno de Primeiro Tipo) nos limites leste e oeste do modelo, impondo um gradiente hidráulico representativo de 0,009.

Figura 2 - Domínio do modelo e condições de contorno de fluxo e transporte empregados nas simulações

Condição de contorno

de carga hidráulica especificada

Condição de contorno

de concenração especificada

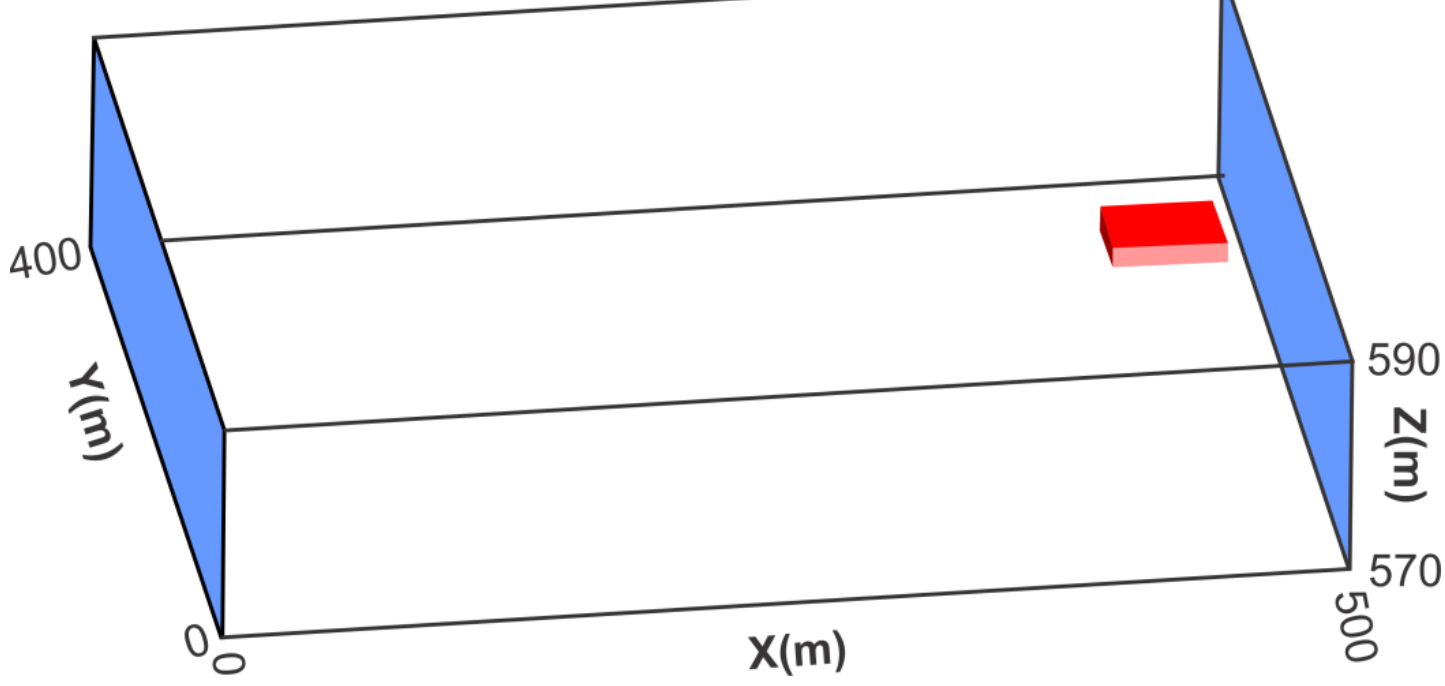

A simulação do transporte de solutos a partir da área-fonte foi conduzida por simulações no MT3DMS (ZHENG \& WANG, 1999).

O emprego de uma condição de contorno de concentração constante para reproduzir uma área-fonte representada por NAPL se baseia no princípio da aproximação de equilíbrio local, que assume a existência de um equilíbrio termodinâmico na interface entre o NAPL e a água (BANERJEE, 1984; SEAGREN et al., 1999; GRANT \& GERHARD, 2007).

Para a área-fonte considerou-se um volume de 5000 m³ $^{3}$ com concentração constante de 1,5 mg/L; os valores de dispersividade longitudinal, transversal e vertical empregados foram 1,0 $\mathrm{m}, 0,05 \mathrm{~m}$ e 0,00001 $\mathrm{m}$, respectivamente.

\section{RESULTADOS}

\subsection{Abundância e distribuição das litofácies}

A distribuição espacial das informações litológicas das 58 sondagens utilizadas está ilustrada na Figura 3. 


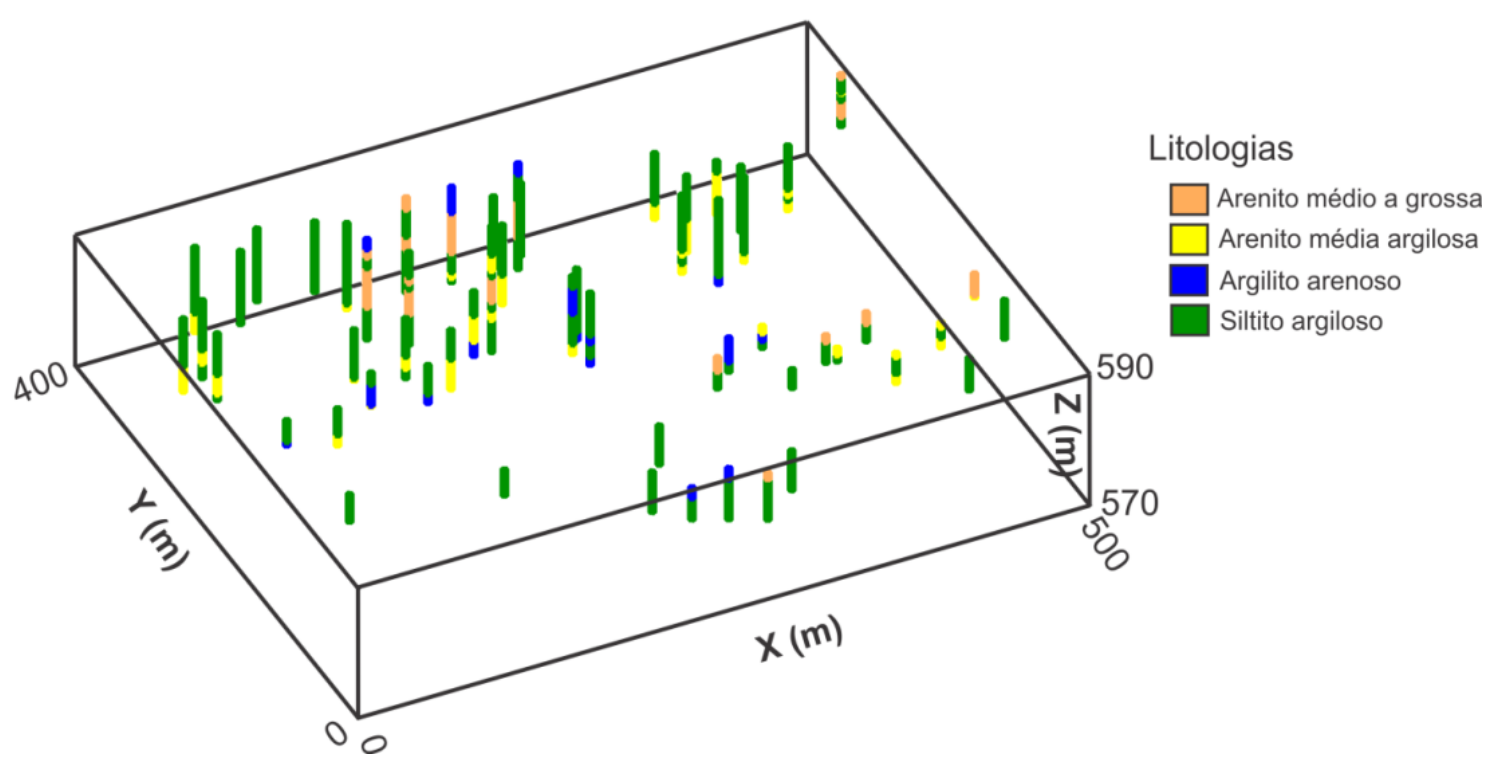

A abundância relativa de litofácies foi estimada a partir do cálculo da porcentagem das espessuras totais de cada litotipo em relação ao conjunto total de informações levantadas. A avaliação da proporção de cada uma das litofácies no conjunto de dados empregado revela o franco predomínio do litotipos pertencentes às fácies de planícies de inundação, que perfazem 79\% do conjunto de dados, enquanto que as litologias arenosas perfazem $21 \%$.

\subsection{Modelos estocásticos}

Cenários geológicos gerados por T-PROGS

A partir da sucessão vertical de litologias identificadas nas sondagens, foram geradas curvas de probabilidade de transição ( $F i$ gura 4) entre as litologias identificadas nas sondagens. Observase que as maiores probabilidades de transição ocorrem da litologia argila arenosa para silte argiloso e areia média a grossa para silte argiloso.

Figura 4 - Matriz de probabilidade de transição ajustada pelas Cadeias de Markov com espaçamento discreto de 0,3 m
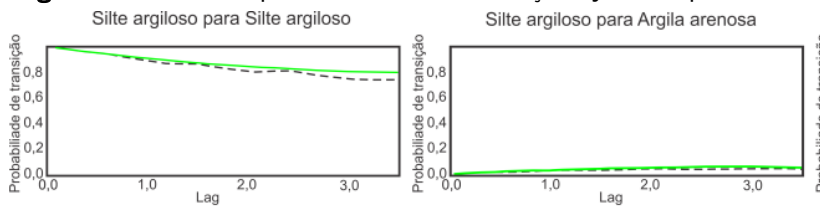

Silte argiloso para Areia média argilosa Silte argiloso para Areia média a grossa
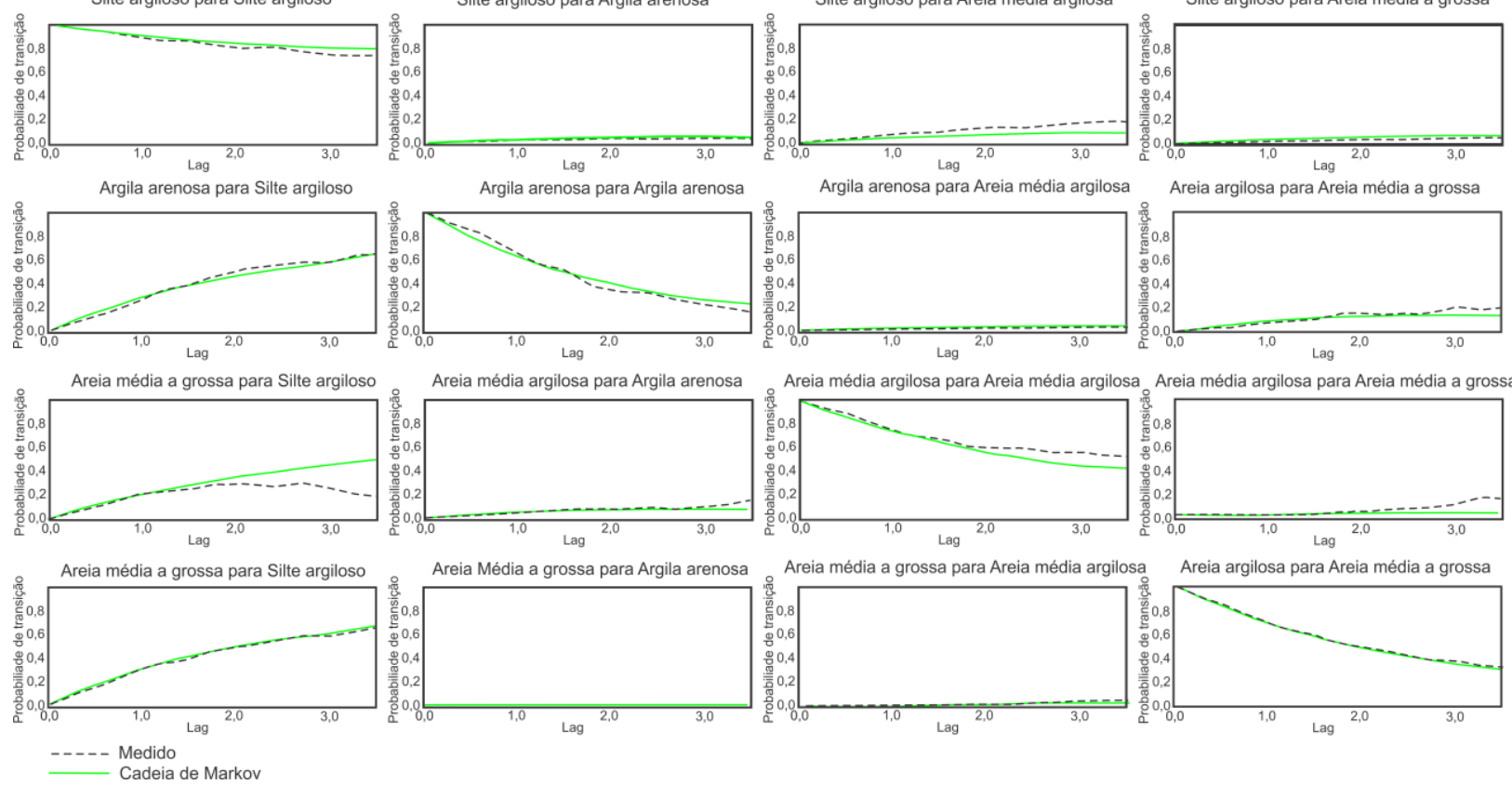

Os cenários simulados pelo T-PROGS apresentaram resultados com elevada variabilidade quanto à distribuição e geometria das fácies.

Para ilustrar o elevado grau de dissimilaridade dos resultados gerados, a Figura 6 apresenta a comparação de dois cenários distintos. 
Figura 5 - Distribuição tridimensional das litofácies em dois cenários distintos
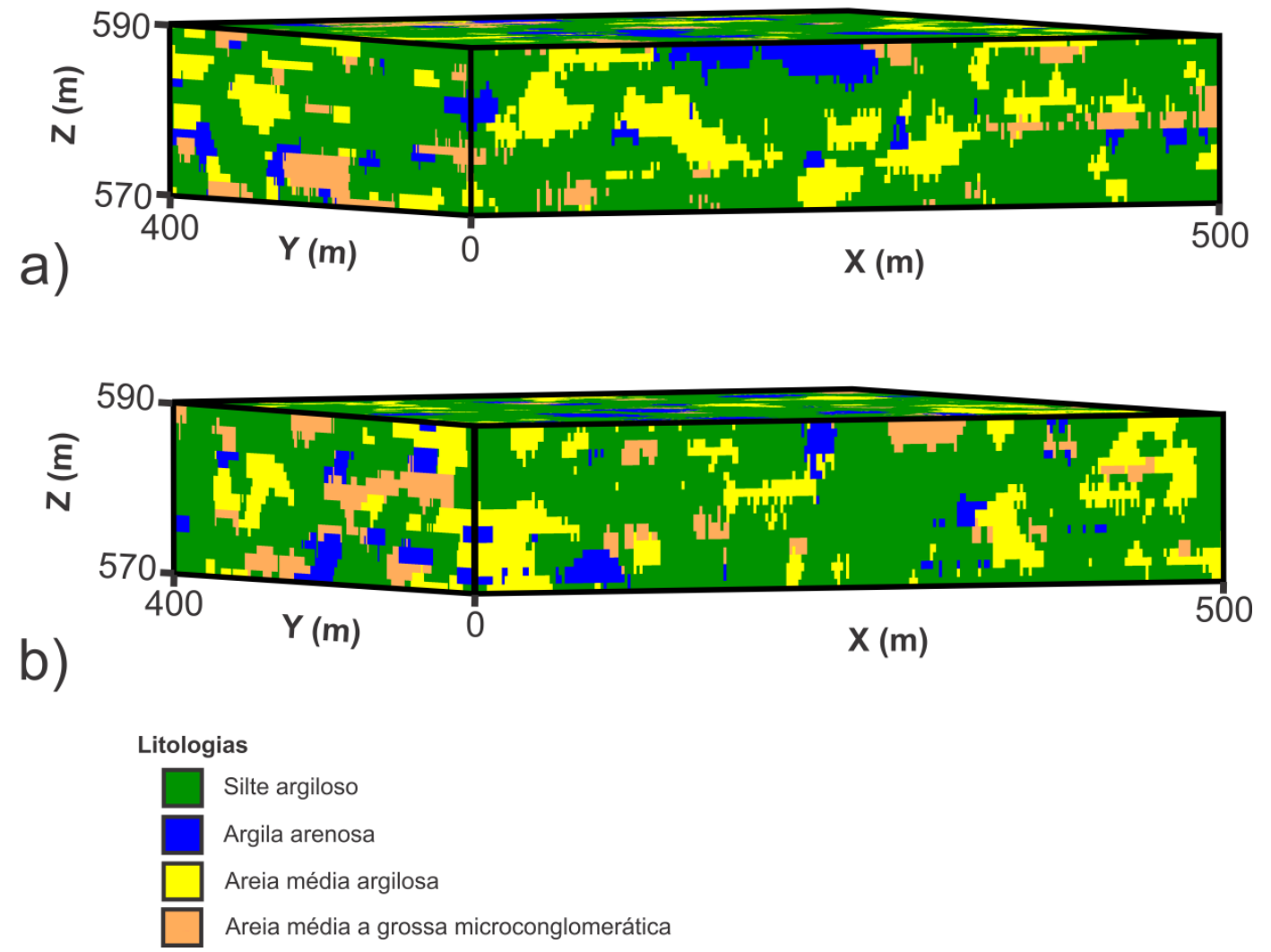

A distribuição das lentes arenosas - representadas pelos litotipos arenito médio a grosso e arenito médio argiloso - nesses 10 cenários é apresentada na Figura 6.

As simulações geraram modelos geológicos caracterizados por baixa conectividade dos corpos arenosos (fácies de canal). Embora com padrões variados, as simulações mostraram predomínio de corpos alongados na direção SE-NW, com espessuras variando entre $1 \mathrm{~m}$ e $5 \mathrm{~m}$. Os corpos arenosos descontínuos podem ser interpretados como resquícios de barras de canal em meandros abandonados. As variações no grau de conectividade dos litotipos arenosos são intrínsecas a ambientes fluviais dominados por fácies de planície de inundação, e as variações na proporção de sedimentos arenosos podem ser interpretadas como variações de fluxo fluvial, dentre outros fatores.

\section{Simulação de transporte}

O uso do aplicativo Visual Modflow mostrou a abrangência tridimensional das plumas de fase dissolvida nos cenários simulados (Figura 7). Os volumes das plumas simuladas variam entre $219.996 \mathrm{~m}^{3}$ (Cenário 7) e $722.620 \mathrm{~m}^{3}$ (Cenário 8), com um valor médio de $487.050 \mathrm{~m}^{3}$ e desvio-padrão de $183.037 \mathrm{~m}^{3}$ (Tabela 2). 
Figura 6 - Distribuição tridimensional dos litotipos arenosos nos 10 cenários simulados (exagero vertical de 10 vezes).
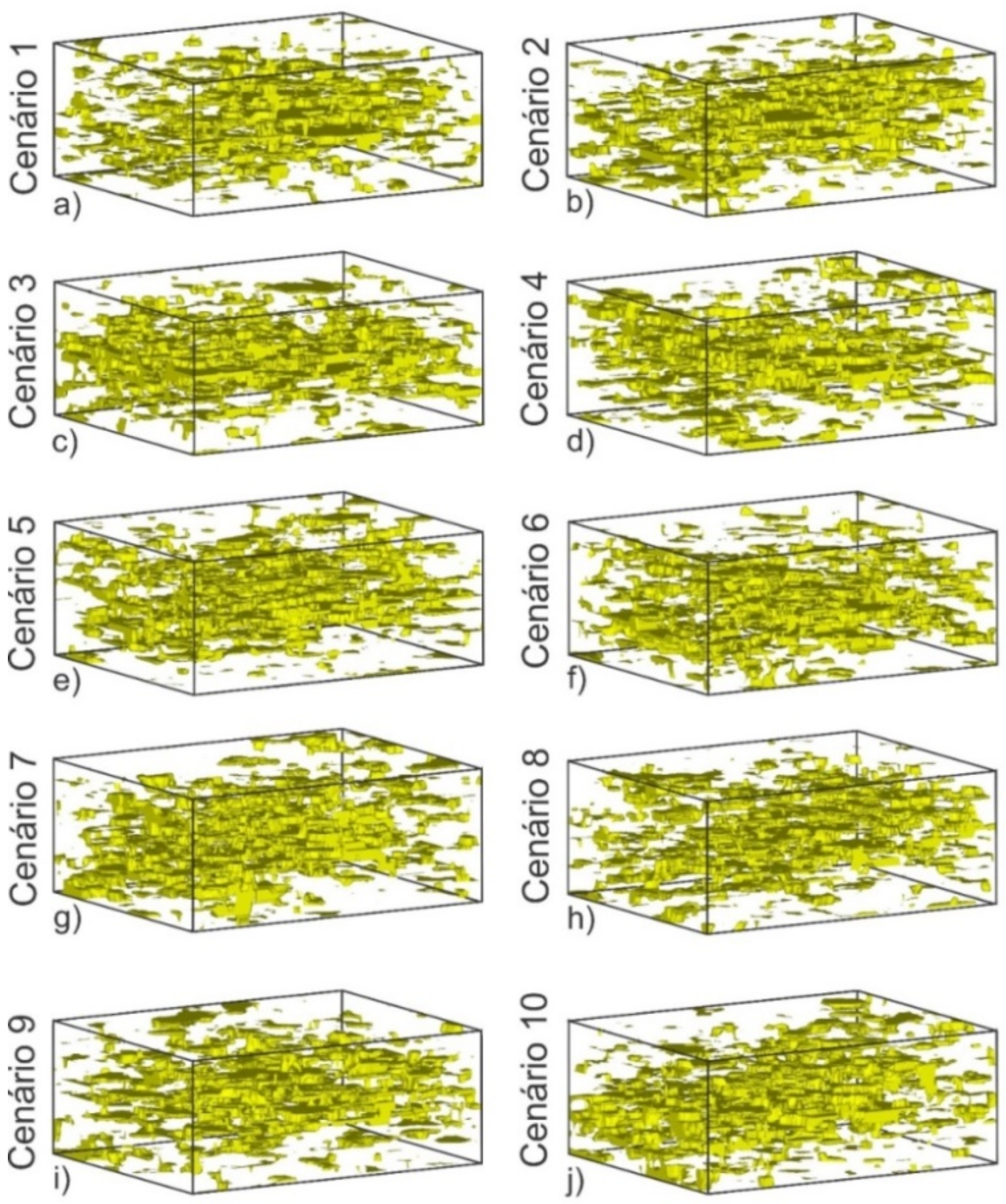
Figura 7 - Representação tridimensional das plumas de fase dissolvida nos 10 cenários gerados pelo T-PROGS (Exagero vertical de 5 vezes)
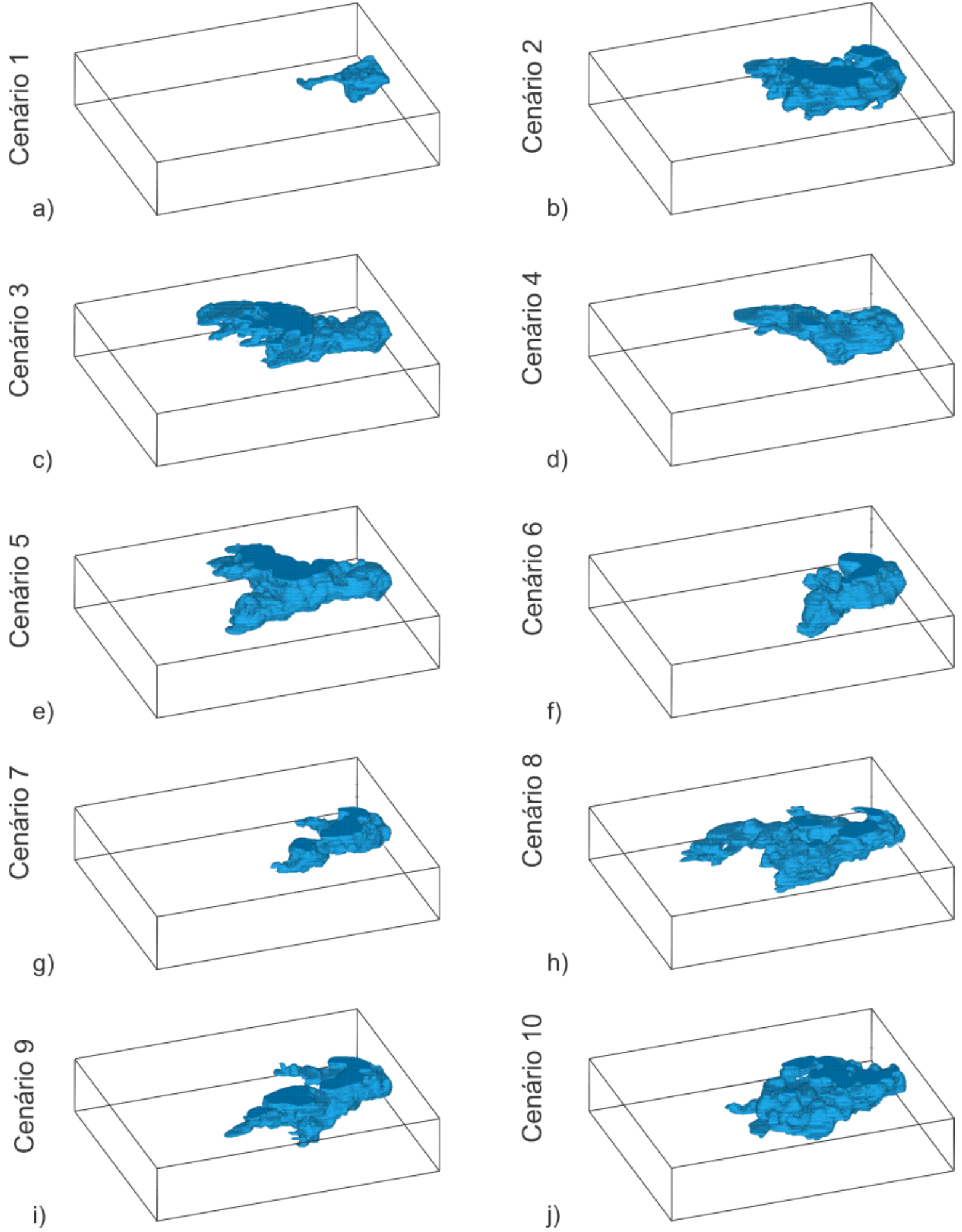
Tabela 2 - Volume das plumas de contaminantes orgânicos dissolvidos, geradas pelas simulações nos 10 cenários simulados

\begin{tabular}{cc}
\hline Simulação & Volume da pluma de fase dissolvida $\left(\mathrm{m}^{3}\right)$ \\
\hline Cenário 1 & 230220 \\
Cenário 2 & 664288 \\
Cenário 3 & 506716 \\
Cenário 4 & 504400 \\
Cenário 5 & 646620 \\
Cenário 6 & 280652 \\
Cenário 7 & 219996 \\
Cenário 8 & 722620 \\
Cenário 9 & 522208 \\
Cenário 10 & 572784 \\
\hline
\end{tabular}

Além da ampla variação volumétrica das plumas simuladas, é possivel notar que as concentrações dentro das plumas de fase dissolvida ostentam fortes variações. A Figura 8 ilustra essas variações dentro das plumas de fase dissolvida a 6 metros de profundidade, onde se encontra a fonte de contaminação nos 10 cenários simulados.

\section{DISCUSSÃO}

As simulações com o T-PROGS geraram cenários geológicos de padrões variados (Figura 5), com predomínio de corpos arenosos descontínuos. As variações de geometria e distribuição espacial dos litotipos arenosos concorreram para que ocorressem volumes e direções de migração bastante variáveis nas simulações de transporte das plumas de fase dissolvida (Figura 7). Tendo em vista que a dispersão hidrodinâmica da pluma é resultado da variabilidade espacial dos valores de $\mathrm{K}$, as heterogeneidades na área de estudo resultam em elevados valores de macrodispersão (Figuras 7 e 8).

Esses resultados indicam baixa conectividade entre os corpos arenosos e sua influência na dispersão da pluma contaminante. Dessa forma, investigações em aquíferos análogos ao aqui descrito devem focar na avaliação do grau de conectividade das litologias mais permeáveis. Esta etapa é particularmente importante em aquíferos marcados pelo predomínio de litotipos sedimentares dotados de baixa permeabilidade, em que a conectividade dos corpos arenosos é restrita.

Modelos numéricos normalmente não apresentam solução única, tendo em vista que um mesmo conjunto de dados de campo pode subsidiar interpretações e modelos conceituais amplamente divergentes entre si, que conduzem à elaboração de diferentes modelos de fluxo. Desse modo, a simples calibração do modelo determinístico não assegura que este seja capaz de fornecer previsões confiáveis. Por essa razão, os modelos estocásticos podem ser empregados como ferramentas complementares para avaliar o grau de incerteza decorrente da heterogeneidade geológica observada e da quantidade de informações disponíveis.

Em razão da elevada variabilidade dos resultados obtidos pelas simulações no presente trabalho, tem-se um elevado grau de incerteza no sítio estudado, sugerindo que as informações geológicas disponíveis são insuficientes para caracterizar adequadamente as heterogeneidades geológicas do aquífero. Contudo, destaca-se que a insuficiência de informações é uma condição generalizada na investigação de áreas contaminadas, em razão da disponibilidade limitada de recursos para investigações ambientais. Nessas investigações, os esforços são normalmente direcionados para delimitação da área-fonte ou das plumas de fase dissolvida, negligenciando-se investigações adicionais para caracterização geológica de detalhe. Por sua vez, as técnicas convencionais de investigação em geral não proporcionam uma caracterização geológica satisfatória. Assim, o emprego de ferramentas alternativas que permitam a aquisição de informações com elevado grau de detalhe e com tempo de investigação menor, tais como a tomografia elétrica ou caracterização de alta resolução, constituem opções viáveis para a caracterização geológica.

É interessante mencionar que os resultados obtidos apontam para um grau de incerteza similar aos demonstrados por Koltermann \& Gorelick (1996), Maxwell et al. (2008), Zhang et al. (2013), Fogg \& Zhang (2016) e Tyukhova \& Willmann (2016). 
Figura 8 - Plumas de fase dissolvida à profundidade de 6 metros, para os 10 cenários simulados (900 dias de simulação)
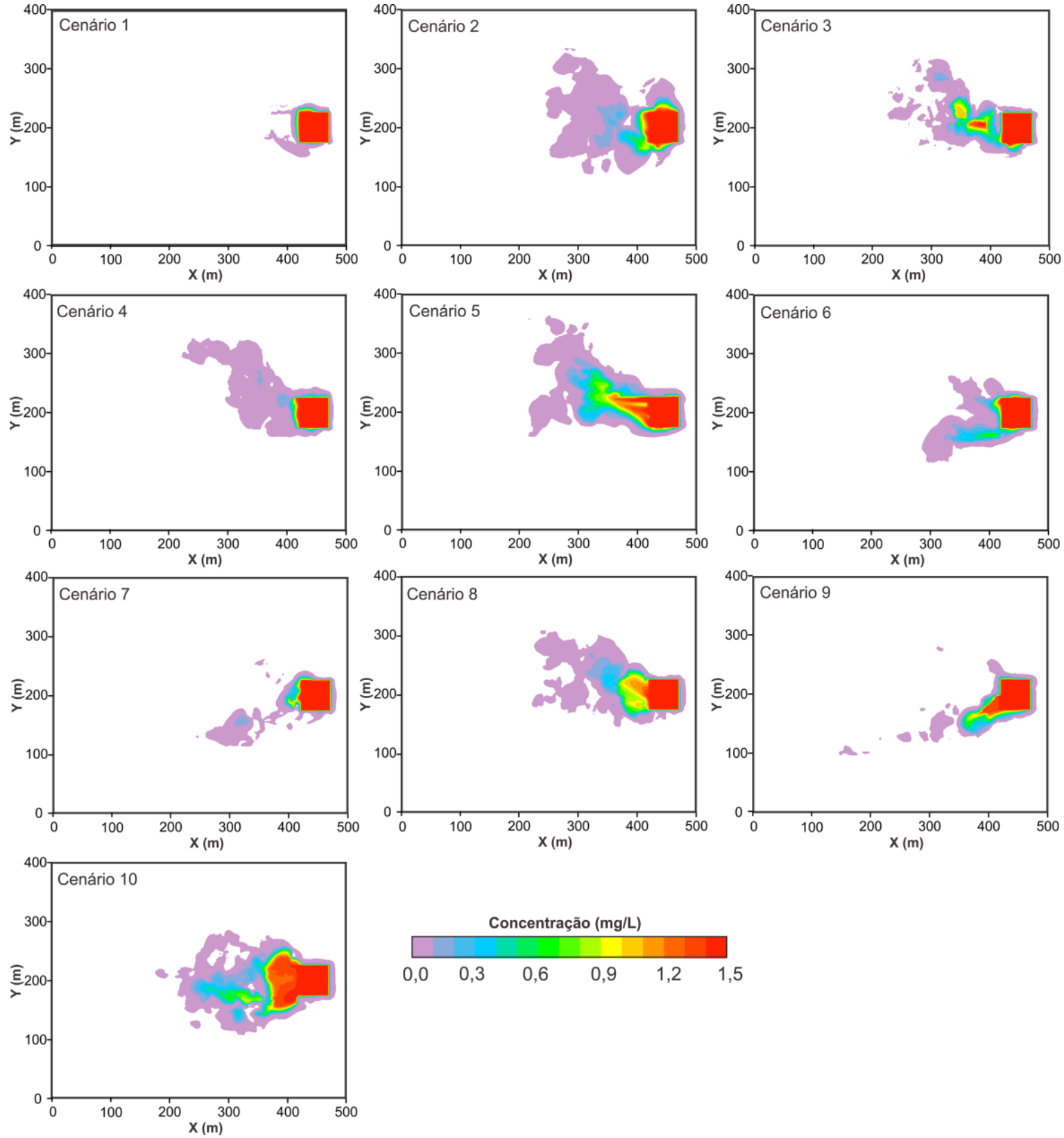

\section{CONCLUSÃO}

As heterogeneidades geológicas continuam representando um grande desafio para a confiabilidade dos modelos numéricos na previsão do comportamento de contaminantes na fase aquosa.

A discrepância observada nas dimensões e na direção de migração da pluma de contaminação indica a existência de fortes incertezas, inerentes à heterogeneidade do aquífero estudado.

A análise comparativa das plumas e da distribuição dos corpos arenosos indica que o transporte de contaminantes dissolvidos em águas subterrâneas é fortemente condicionado pela continuidade e conectividade de litotipos arenosos em aquíferos de origem fluvial com domínio de fácies pelíticas. Tendo em vista que as informações geológicas normalmente são insuficientes para identificar adequadamente a geometria dos corpos arenosos, modelos para prever a migração de contaminantes dissolvidos na água estão sujeitos a elevados graus de incerteza. Essa constatação sugere que as investigações geológicas devem detalhar a geometria e arquitetura dos litotipos, com vistas à elaboração de modelos numéricos mais consistentes, a partir da redução de incertezas. De maneira similar, este critério deve ser utilizado para o planejamento dos sistemas de remediação a serem implantados.

\section{REFERÊNCIAS}

BANERJEE, S. Solubility of organic mixtures in water. Environmental Science \& Technology, v. 18, n. 8, p. 587-591, 1984. Disponível em: $<10.1021 /$ es00126a004>. Acesso em: 12 mar. 2017.

BIANCHI, M.; ZHENG, C.; WILSON, C.; TICK, G.R.; LIU, G.; GORELICK, S. M. Spatial connectivity in a highly heterogeneous aquifer: From cores to preferential flow paths. Water Resources Research, v. 47, n. 5, 2011. Disponivel em: < 10.1029/2009WR008966>. Acesso em: 13 mar. 2017.

CARLE, S.F.; FOGG, G.E. Modeling spatial variability with one and multidimensional continuous-lag Markov chains. Mathematical Geology, v. 
29, n. 7, p. 891-918, 1997. Disponível em: < 10.1023/A:1022303706942>. Acesso em: 13 mar. 2017.

FERNANDES, A. J. Tectônica Cenozóica na Porção Media da Bacia do rio Piracicaba e sua Aplicação à Hidrogeologia. Tese (Doutoramento em Recursos Minerais e Hidrogeologia). Instituto de Geociências, Universidade de São Paulo. São Paulo, 244 F, 1997.

FOGG, G.E. Groundwater flow and sand body interconnectedness in a thick, multiple-aquifer system. Water Resources Research, v. 22, n. 5, p. 679-694, 1986. Disponível em: <10.1029/WR022i005p00679>. Acesso em: 13 mar. 2017.

FOGG, G. E.; ZHANG, Y. Debates-Stochastic subsurface hydrology from theory to practice: A geologic perspective. Water Resources Research 2016. Disponível em: <10.1002/2016WR019087>. Acesso em: 16 maio 2017.

GELHAR, L. W. Stochastic subsurface hydrology from theory to applications. Water Resources Research, v. 22, n. 9S, 1986. Disponivel em: <10.1029/WR022i09Sp0135S>. Acesso em: 12 mar. 2017

GRANT, G. P.; GERHARD, J. I. Simulating the dissolution of a complex dense nonaqueous phase liquid source zone: 2 . Experimental validation of an interfacial area-based mass transfer model. Water Resources Research, v. 43, n. 12, 2007. Disponível em: <10.1029/2007WR006038. >. Acesso em: 11 mar. 2017.

HARBAUGH, A.W., MODFLOW-2005, the U.S. Geological survey modular ground-water model: the Ground-Water Flow Process. U.S. Geological Survey Techniques and Methods 6-A16. 2005.

KOLTERMANN, C.E.; GORELICK, S.M. Heterogeneity in sedimentary deposits: A review of structure-imitating, process-imitating, and descriptive approaches. Water Resources Research, v. 32, n. 9, p. 2617-2658, 1996. Disponível em: <10.1029/96WR00025>. Acesso em: 13 mar. 2017.

KRUMBEIN, W. C.; DACEY, M. F. Markov chains and embedded Markov chains in geology. Mathematical Geology, v. 1, n. 1, p. 79-96, 1969. Disponível em: <10.1007/BF02047072>. Acesso em: 13 mar. 2017

MAXWELL, R.M.; CARLE, S.F.; TOMPSON, A.F.B. Contamination, risk, and heterogeneity: on the effectiveness of aquifer remediation. Environmen tal Geology, v. 54, n. 8, p. 1771-1786, 2008. Disponível em: <10.1007/s00254-007-0955-8>. Acesso em: 13 mar. 2017.
MELO, M.S. A Formação Rio Claro e depósitos associados: sedimentação neocenozóica na Depressão Periférica Paulista, São Paulo, 144 F. Tese de Doutorado, IG-USP, 1995.

MORENO, L.; TSANG, C. F. Flow channeling in strongly heterogeneous porous media: A numerical study. Water Resources Research, v. 30, p. 1421-1421, 1994. Disponível em: < 10.1029/93WR02978>. Acesso em: 13 mar. 2017.

SEAGREN, E. A.; RITTMANN, B. E.; VALOCCHI, A.J. An experimental investigation of NAPL pool dissolution enhancement by flushing. Journal of Contaminant Hydrology, v. 37, n. 1, p. 111-137, 1999. Disponível em: < doi.org/10.1016/S0169-7722(98)00157-0>. Acesso em: 10 mar 2017.

TERAMOTO, E. H. Estudo da efetividade da atenuação natural de compostos BTEX em área contaminada por querosene de aviação. Instituto de Geociências e Ciências Exatas, Universidade Estadual Paulista, Rio Claro. 205 f. 2015.

TERAMOTO, E. H.; CHANG, H. K. Field data and numerical simulation of btex concentration trends under water table fluctuations: Example of a jet fuel-contaminated site in Brazil. Journal of Contaminant Hydrology, v. 198, p. 37-47, 2017. Disponivel em: <10.1016/j.jconhyd.2017.01.002>. Acesso em: 01 mar. 2017.

TYUKHOVA, A.R.; WILLMANN, M. Conservative transport upscaling based on information of connectivity. Water Resources Research, v. 52, n. 9, p. 6867-6880, 2016. Disponível em: <10.1002/2015WR018331>. Acesso: 16 maio 2017

ZHANG, Y.; GREEN, C. T.; FOGG, G. E. The impact of medium architecture of alluvial settings on non-Fickian transport. Advances in Water Resources, v. 54, p. 78-99, 2013. Disponível em: <doi.org/10.1016/j.advwatres.2013.01.004>. Acesso em: 15 mar. 2017.

ZHENG, C. E WANG, P. MT3DMS: a modular three-dimensional multispecies transport model for simulation of advection, dispersion, and chemical reactions of contaminants in groundwater systems. Documentation and User's Guide, US Army Corps of Engineers Engineer Research and Development Center (1999). 